\title{
Evaluación de la calidad del aire en el establo de bovinos de la estación experimental de Chachapoyas de la Universidad Nacional Toribio Rodríguez de Mendoza de Amazonas, 2017
}

\section{Evaluation of the air quality in the bovine stable of the Chachapoyas experimental station of the Toribio Rodriguez de Mendoza National University of Amazonas,}

\author{
Fernando Isaac Espinoza Canaza, Julio Mariano Chávez Milla ${ }^{2}$
}

\begin{abstract}
RESUMEN
La investigación tuvo como objetivo estudiar la presencia de metano y la calidad el aire en los núcleos donde se albergan los bovinos localizados en el Centro Experimental de Chachapoyas, y su afectación en la salud de las personas que laboran en el cuidado y mantenimiento del ganado. El lugar de estudio está localizado en el campus universitario, en Chachapoyas a $2350 \mathrm{msnm}$ con temperaturas promedio entre $14{ }^{\circ} \mathrm{C}$ a $25{ }^{\circ} \mathrm{C}$ y cuya humedad relativa está entre $60 \%$ y $70 \%$. Para la adquisición de datos se utilizó un sensor de gas metano (CH4) el MQ-4, sensor de temperatura y humedad el DHT11 y un módulo arduino UNO. Los datos se registraron en intervalos de un minuto, para poder captar de forma inmediata la presencia de metano. Los recintos donde se realizó la adquisición de datos fueron en el núcleo 1 localizado el corral principal de bovinos que alberga entre 25 cabezas de ganado, se censó en rangos de 38 ppm a 74 ppm; núcleo 2 que corresponde al recinto de ordeño automatizado que alberga de 6 a 8 cabezas de ganado, se registró entre 45 ppm a 122 ppm y en el núcleo 3 que corresponde al sector de desagüe hacia el biodigestor se registró entre $64 \mathrm{ppm}$ a $364 \mathrm{ppm}$ respectivamente. La concentración de metano por encima de 1000 ppm resultan ser peligrosos para la salud y seguridad de la personas. En conclusión los núcleos 1, 2 y 3 mantienen concentraciones de metano por debajo de los estándares permitidos por lo que los núcleos indicados mantienen el aire saludable.
\end{abstract}

Palabras clave: Pureza de aire, contaminación, gases incoloros, olores.

\begin{abstract}
The objective of the research was to study the presence of methane and air quality in the nuclei where the bovines located in the Chachapoyas Experimental Center are housed, and their impact on the health of the people who work in the care and maintenance of livestock. The place of study is located on the university campus, in Chachapoyas at2350 m.s.n.m. with average temperatures between $14^{\circ} \mathrm{C}$ to $25^{\circ} \mathrm{C}$ and whose relative humidity is between $60 \%$ and $70 \%$. For the acquisition of data, a methane gas sensor (CH4) was used, the MQ-4, a DHT11 temperature and humidity sensor and an module Arduino UNO. The data were recorded in one-minute intervals, in order to immediately capture the presence of methane. The enclosures where the acquisition of data was carried out were in the nuclei 1 located in the main corral of cattle that houses between 25 heads of cattle, it was counted in ranks from $38 \mathrm{ppm}$ to $74 \mathrm{ppm}$; nuclei 2 that corresponds to the automated milking enclosure that houses 6 to 8 heads of cattle, was recorded between $45 \mathrm{ppm}$ to $122 \mathrm{ppm}$ and in nuclei 3 that corresponds to the drainage sector to the biodigester was recorded between $64 \mathrm{ppm}$ to $364 \mathrm{ppm}$ respectively. The concentration of methane above $1000 \mathrm{ppm}$ turns out to be dangerous for the health and safety of people. In conclusion nuclei 1, 2 and 3 maintain methane concentrations below the allowed standards so the indicated nuclei keeps the air healthy.
\end{abstract}

Keywords: Air purity, pollution, colorless gases, odors.

\footnotetext{
Ingeniero Electrónico. Profesor auxiliar, Facultad de Ingeniería de Sistemas y Mecánica Eléctrica, Universidad Nacional Toribio Rodríguez de Mendoza de Amazonas. Correo electrónico fernando.espinoza@untrm.edu.pe

${ }^{2}$ Biólogo, Profesor Asociado, Facultad de Ingeniería de Sistemas y Mecánica Eléctrica, Universidad Nacional Toribio Rodríguez de Mendoza de Amazonas. Correo electrónico julio.chavez@untrm.edu.pe
} 


\section{INTRODUCCIÓN}

Cerca de 500 millones de toneladas métricas/año de metano ingresan a la atmósfera debido a actividades antropogénicas y fenómenos naturales. A esta tasa se espera que el metano cause cerca del 15-17\% del calentamiento global (Carmona, 2005).

Las enfermedades crónicas como la bronquitis también se ve afectada por las alteraciones de la calidad del aire; en este sentido, aumentos en la exposición al ozono se asocian significativamente con crecimientos de $2.1 \%$ en los casos de padecimientos de la bronquitis crónica, resultados que no contradicen los encontrado (Chadwick, 2005), $2.4 \%$ al evaluar los efectos de corto plazo del ozono sobre la enfermedad pulmonar obstructiva crónica en Amsterdam.

La concentración atmosférica de los gases de efecto invernadero ha aumentado a partir de la revolución industrial y particularmente en los últimos 50 años debido a las diversas actividades humanas, sobre todo la utilización de combustibles fósiles y los cambios en el uso de los diferentes tipos de suelos. Lo anterior, ha contribuido a cambios en el clima del planeta (IPCC, 2001). La temperatura de la superficie terrestre y marina ha incrementado, los patrones espaciales y temporales de las precipitaciones han cambiado; se ha elevado el nivel del mar y ha aumentado la frecuencia e intensidad de los fenómenos asociados con El Niño (IPCC, 2001). El cambio climático representa un peligro para los ecosistemas y para la propia sobrevivencia del hombre en el planeta. De allí, la importancia de conocer el papel de algunos ecosistemas como reguladores del ciclo del C en la tierra.

El aire limpio está compuesto principalmente por nitrógeno y oxígeno, en pequeñas proporciones se puede encontrar vapor de agua y dióxido de carbono. La contaminación del aire, proviene de la adición de sustancias emitidas a la atmósfera que causan un desequilibrio en la composición original. El aire contaminado contiene gases, polvos, olores y humos en grandes cantidades que dañan la salud de las personas, animales y plantas. Además deteriora la infraestructura urbana y algunos materiales de uso cotidiano. Estos contaminantes se dividen en primarios, que se emiten directamente a la atmósfera como resultado de un proceso de combustión (como el $\mathbf{S O}_{2}, \mathbf{C O}, \mathbf{P b}$, etc.) y secundarios, cuando reaccionan los contaminantes primarios con otros contaminantes con igual toxicidad $\left(\mathbf{O}_{3}, \mathbf{N O}_{2}\right.$, etc. $)$ (UNAM, 2017).

La producción de $\mathrm{CH}_{4}$ en los rumiantes está influenciada por factores como consumo de alimento, composición de la dieta, digestibilidad del alimento, procesamiento previo del alimento y frecuencia de alimentación. Entre las estrategias para mitigar las emisiones de $\mathrm{CH}_{4}$ se ha propuesto: reducir el número de animales rumiantes, aumentar el número de animales no rumiantes, manipulación genética de los microorganismos ruminales metanogénicos, desarrollo de razas menos m e tanogénicas y mani pulación dietética-nutricional; esta última parece ser la de mayor potencial en términos de simplicidad y factibilidad. La manipulación nutricional para suprimir la metanogénesis incluye uso de forrajes de alta calidad, alta proporción de granos en la dieta, uso de aditivos (compuestos químicos, ácidos orgánicos, ionóforos, probióticos), dietas ricas en ácidos grasos insaturados, adición de acetógenos, de bacteriocinasas, de virus vs Archaea, y de extractos vegetales (aceites esenciales), modificación de las prácticas de alimentación y suplementación a dietas basadas en pajas. Estas prácticas de alimentación reducen las emisiones de $\mathrm{CH}_{4}$ por la modificación de la fermentación ruminal, inhibiendo directamente los metanogénicos y protozoarios, o desviando los iones hidrógeno de los metanogénicos (Bonilla, 2012).

El metano $\left(\mathrm{CH}_{4}\right)$ se produce fundamentalmente por la descomposición anaerobia de la materia orgánica en los sistemas biológicos. Los procesos agrícolas como el cultivo del arroz inundado en agua, la fermentación entérica en los animales y la descomposición de los desechos de éstos, y la descomposición de los desechos municipales emiten $\mathrm{CH}_{4}$. El CH $\mathrm{CH}_{4}$ también se emite durante la producción y distribución de gas natural y petróleo, y es liberado como subproducto en la extracción del carbón y en la combustión incompleta de los energéticos fósiles (Cuatecontzi y Gasca, 2004).

El objetivo fue evaluar la calidad del aire los núcleos seleccionados del centro del establo de bovinos localizado en la estación experimental de Chachapoyas de la Universidad Nacional Toribio Rodríguez de Mendoza de Amazonas, específicamente el nivel de concentración de metano $\left(\mathrm{Ch}_{4}\right)$.

La metodología a considerar fue de implementar un sistema de sensores detectores de gases contaminantes particularmente del metano $\left(\mathrm{Ch}_{4}\right)$, medir la temperatura y la humedad relativa para poder evaluar la calidad del aire en tres recintos del establo: El área de ordeño (núcleo 2), el área o zona de comedero de los animales (núcleo 1) y el área del biodigestor (núcleo 3). 


\section{MATERIAL Y MÉTODOS}

El área de estudio se llevó acabo en la Estación Experimental de Chachapoyas (establo) localizado en el Campus de la Universidad Nacional Toribio Rodríguez de Mendoza a 2230 m.s.n.m.

\section{Metodología}

El tipo de investigación realizada es de carácter experimental, consistente en medir el nivel de concentración de metano $\left(\mathrm{CH}_{4}\right)$ en los núcleos 1,2 y 3 respectivamente.

\section{Materiales y procedimientos}

La población de bovinos que genera el gas metano estuvo constituido entre 25 bovinos, de acuerdo al siguiente detalle: 07 vacas lecheras de la raza Jersey (productoras), 07 vacas lecheras de la raza Jersey (en seca), 09 terneras de la raza Jersey y 02 becerros de la raza Angus y Jersey.

Para el estudio se tomó al $100 \%$ de la población de bovinos, en razón de que comparten el mismo recinto para la dieta alimenticia, el estiércol acumulado es global, el espacio para el desplazamiento es común para todos.

\section{Núcleos de estudio}

Núcleo 1: El recinto donde están ubicados todos los animales. Este es un recinto acondicionado adecuadamente con techo elevado, con bastante ventilación y espacio suficiente para el desplazamiento de los bovinos, es una zona donde los animales comen, beben agua, descansa y excretan.

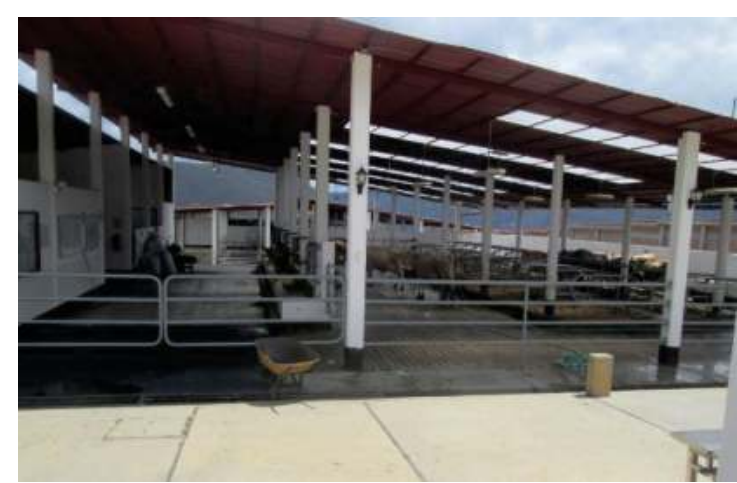

Figura 1: Recinto del núcleo 1

Núcleo 2: Es el área donde se realizad el proceso de ordeño automatizado. Es un recinto acondicionado adecuadamente, no tiene suficiente ventilación, es un espacio diseñado para ordeñar 8 vacas en simultáneo.

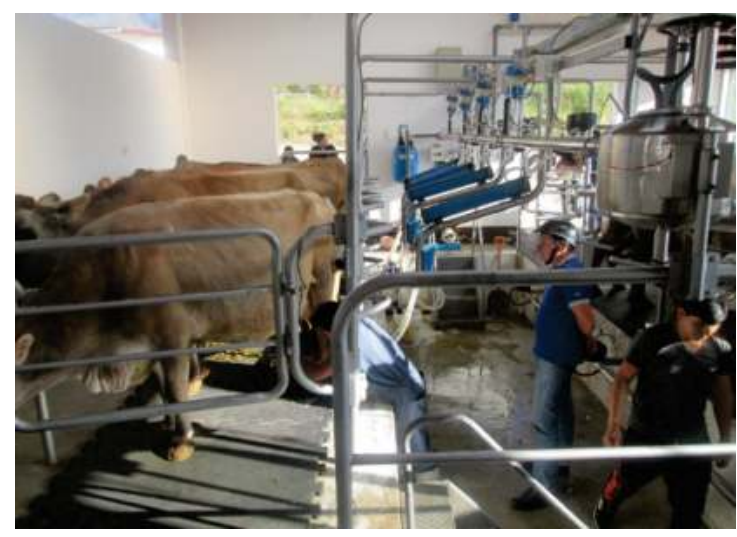

Figura 3: Recinto del núcleo 2

Núcleo 3: Es la zona de desagüe que recolecta los residuos de estiércol provenientes de los bovinos del núcleo 1 ; canalizado para que alimente el pozo donde está instalado un biodigestor.

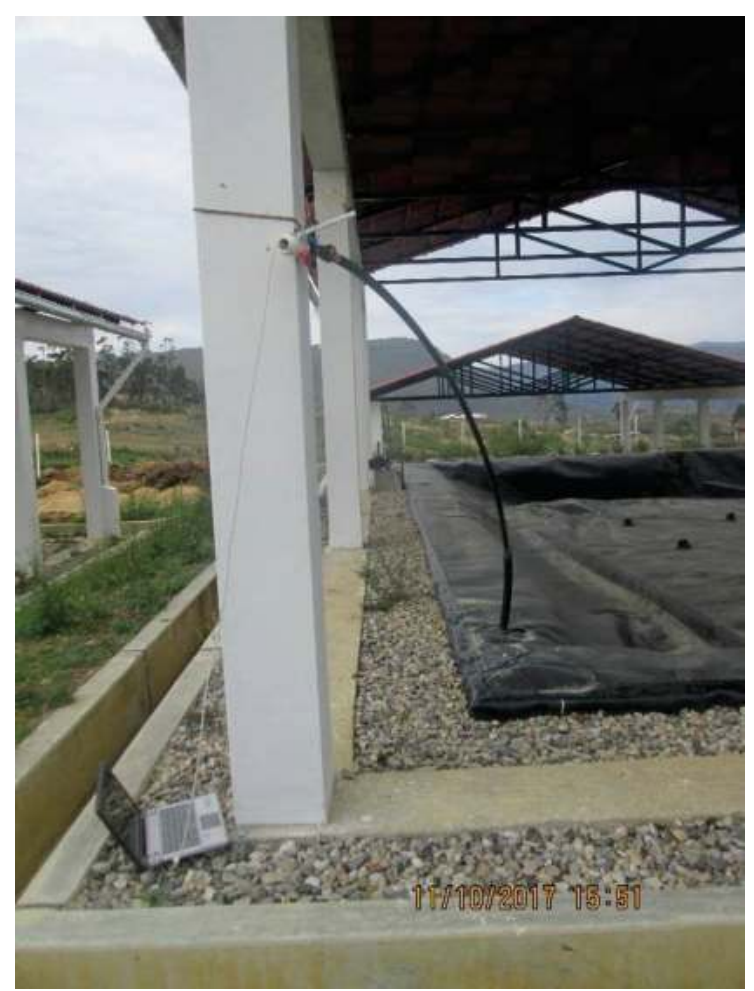

Figura 2: Recinto del núcleo 3

Equipos necesarios para la adquisición y procesamiento de datos

Un sensor de gas metano de la serie MQ-4 para la adquisición de datos, un módulo Arduino UNO para el registro de datos, un sensor DH11 para el registro de la temperatura del medio ambiente y la humedad relativa y una computadora personal para el almacenamiento y procesamiento de datos. 


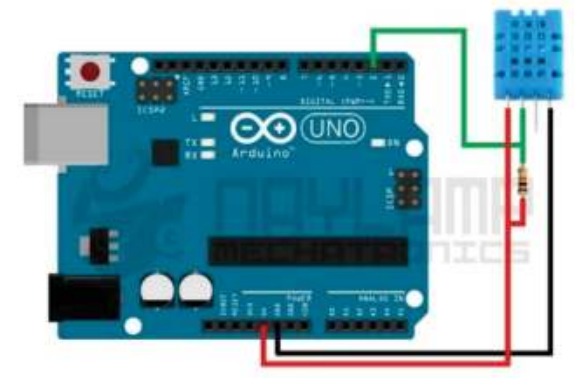

Figura 4: Esquema de conexión del DHT11

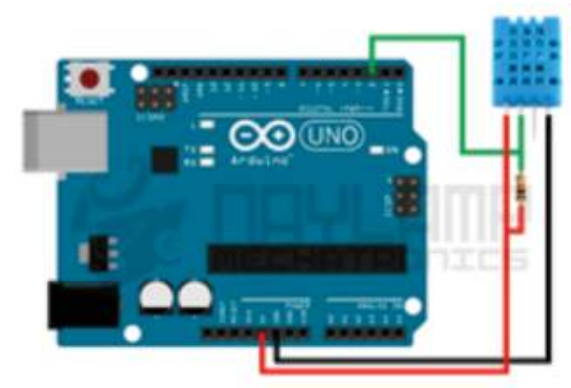

Figura 5: Esquema de conexión del sensor MQ-4

\section{Registro de datos}

Para la adquisición y lectura de datos, se utilizó el módulo de Arduino UNO, que están basada en el chip ATmega328, que tiene 14 pines de entrada/salida (E/S) digitales, de los cuales 6 puedan simular salidas análogas mediante la técnica PWM y 6 entradas análogas. Funciona a una velocidad de $16 \mathrm{Mhz}$ y tiene $32 \mathrm{~Kb}$ de memoria flash y $2 \mathrm{~Kb}$ de memoria SRAM, implementándose conforme se muestra en las Figuras 7 y 8.

El registro de datos se llevó acabo cada 10 segundos, se consideró dicho tiempo tomando en cuenta que el gas metano es menos denso que el aire y es altamente volátil por lo tanto el tiempo de detección del gas tuvo que ser lo más corto posible.

\section{Procedimiento para la recolección de datos}

Para el núcleo 1, se tomó en cuenta la totalidad de la población bovina de 25 , en el periodo de ingesta de alimentos; se activa el sensor de metano MQ-4 y se inicia el registro automático de datos directamente a la computadora.

Para el núcleo 2, se realizó en horas de la tarde entre las 4:30 pm a 5:00 pm, hora de ordeño con la población de 6 a 7 bovinos.
Para el núcleo 3, se realizó en el periodo de limpieza de las excretas de los animales que están en el núcleo 1; para la medición de los niveles de metano, el sensor se colocó en la compuerta que alimenta al biodigestor.

\section{RESULTADOS}

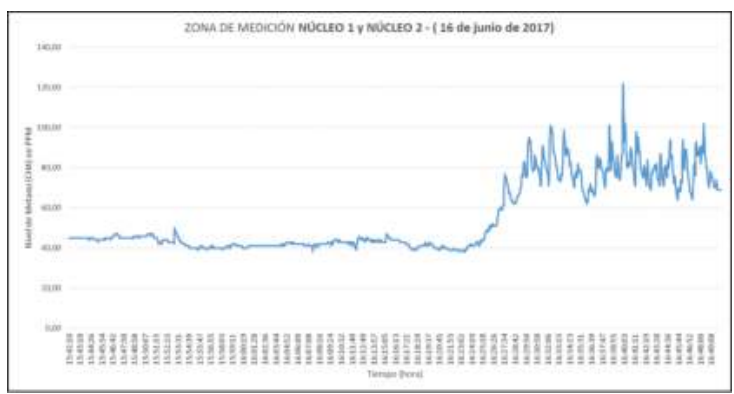

Figura 6. Medición de metano en los ámbitos del núcleo 1 y núcleo 2.

Los resultados obtenidos en la Figura 6, corresponden a dos ámbitos de estudio: núcleo 1 (recinto principal que alberga a los 25 bovinos) y núcleo 2 (recinto para el proceso de ordeñado automatizado).

De acuerdo a la gráfica se puede observar el cambio abrupto que tiene el nivel de concentración de metano al pasar del núcleo 1 al núcleo 2; siendo estos valores en el núcleo 1 entre 38 ppm - 43 ppm y en el núcleo 2 la concentración de metano está entre 45 ppm - $122 \mathrm{ppm}$. El entorno ambiental registró una temperatura ambiente promedio de $22{ }^{\circ} \mathrm{C}$ y una humedad relativa de $59 \% \mathrm{Hr}$.

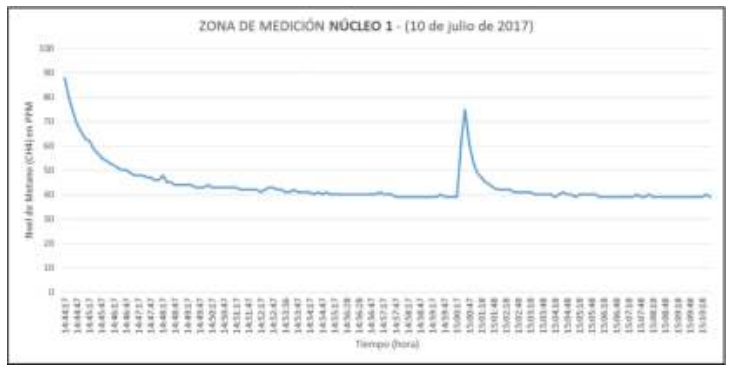

Figura 7. Medición de metano en los ámbitos del núcleo 1.

Los resultados obtenidos en la Figura 7, corresponden a dos ámbitos de estudio: núcleo 1 (recinto principal que alberga a los 25 bovinos).

En el intervalo de tiempo entre las 14:44:47 y 14:52:17, se puede observar que el nivel de metano es relativamente alto y desciende uniforme de $88 \mathrm{ppm}$ a $39 \mathrm{ppm}$. 
En entorno ambiental, registró una temperatura ambiente promedio entre 20 y $21^{\circ} \mathrm{C}$ y una humedad relativa que oscila entre 60 y $62 \% \mathrm{Hr}$.

En el intervalo de tiempo entre las 15:00:07 y 15:02:18, se nota un abrupto y brusco incremento del nivel de concentración de metano emanado por la población bovina del recinto, ya que se eleva de 39 ppm a 75 ppm.

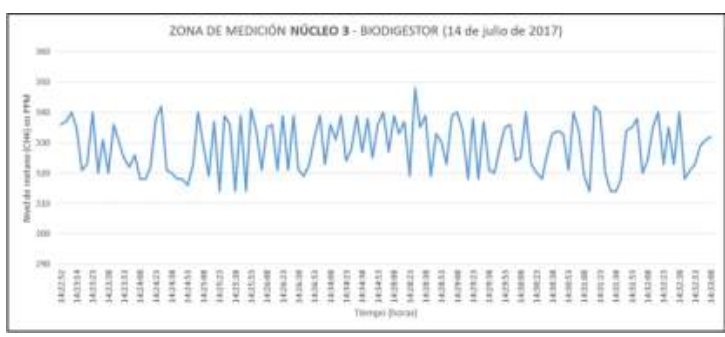

Figura 9. Medición de metano en los ámbitos del núcleo 3 -Biodigestor.

Los resultados obtenidos en la Figura 9, corresponden a dos ámbitos de estudio: núcleo 3 (en el biodigestor).

El intervalo de tiempo entre las 14:22:52 y 14:33:08, se puede observar los niveles concentración de metano es variante en un reducido margen llegando a niveles de concentración pico entre 314 ppm y 348 ppm y se mantiene dentro de ese rango.

Esto puede explicarse por el proceso anaeróbico que está ocurriendo en el en la cámara del biodigestor. Se registró una temperatura ambiente promedio de 20 ${ }^{\circ} \mathrm{C}$ y una humedad relativa entre 65 y $66 \% \mathrm{Hr}$.

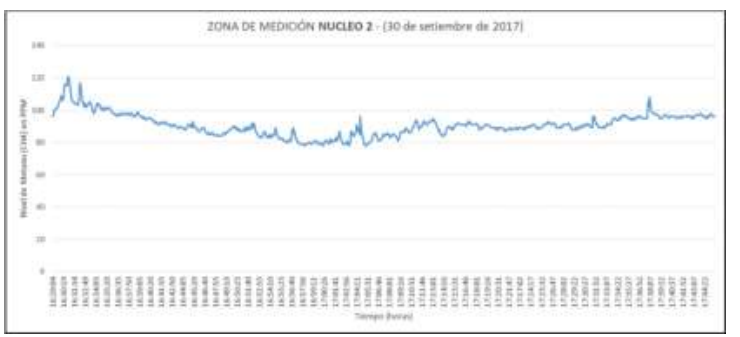

Figura 10. Medición de metano en los ámbitos del núcleo 2.

Los resultados obtenidos en la Figura 10, corresponden a dos ámbitos de estudio: núcleo 2 .

El intervalo de tiempo entre las 16:29:04 y 17:44:22 corresponde al núcleo 2, y se puede observar claramente que el nivel de metano presenta variaciones y de elevada concentración dentro de los límites entre 78 ppm y 119 ppm, esto se debe porque dicho recinto es reducido, casi cerrado y de poca ventilado, en este núcleo, en el intervalo de tiempo indicado se está realizando el proceso de ordeño de 5 vacas y su nivel de concentración de metano puede darse por los gases que emanan de los 5 bovinos.

Se registró una temperatura ambiente promedio de 22 ${ }^{\circ} \mathrm{C}$ y una humedad relativa entre 62 y $63 \% \mathrm{Hr}$.

De acuerdo a los resultados mostrados en las Figuras $6,7,8,9$ y 10 se pueden observar que los niveles de concentración de metano $\left(\mathrm{Ch}_{4}\right)$, es muy variante debido a la volatilidad del gas ya que es menos denso que el aire.

\section{DISCUSIÓN}

En el estudio de investigación realizado, se determinó que en todos los recintos de bovinos en los núcleos 1 , 2 y 3 de la estación experimental Chachapoyas, emanan metano en distintos niveles de concentración, esta producción es por la ingesta de 13 kilos de heno 38 kilos de ensilado (maíz chala triturado) 7 kilos de concentrado (maíz, soya, afrecho, polvillo, harina de pescado, aceite, bicarbonato de sodio, sal común y mineral); para un total de 58 kilos por cada intervalo de alimentación.

Según, Montenegro, (2000), indica que los bovinos poseen un sistema digestivo que tiene la capacidad de aprovechar y convertir material fibroso con altos contenidos de carbohidratos estructurales, en alimentos de alta calidad nutritiva, la carne y la leche. Sin embargo por sus características innatas, este mismo sistema digestivo también produce metano, un potente gas con efecto invernadero que contribuye con aproximadamente el $18 \%$ del calentamiento global ocasionado por actividades productivas con animales domésticos, superado sólo por el $\mathrm{CO}_{2}$.

El metano es producido por microorganismos del rumen durante la fermentación anaeróbica de carbohidratos solubles y estructurales principalmente, siendo estos últimos preponderantes en dietas basadas en forrajes (Kurihara, 1999).

El gas metano constituye aproximadamente el 16 $\%$ de las emisiones mundiales de gases de efecto invernadero. El metano es emitido por una variedad de fuentes tanto antropogénicas (causada por las actividades del ser humano) como de fuentes naturales (Methane, 2008).

Si existe la posibilidad de exposición superior a 1000 ppm, utilice un equipo de respiración con suministro de aire y máscara completa, aprobado por el NIOSH, en modo de presión a demanda $\mathrm{u}$ otro modo de presión positiva. Para aumentar la protección, 
utilícelo en combinación con un equipo de respiración autónomo o cilindro de aire para escape de emergencia. La exposición a 5000 ppm constituye un peligro inmediato para la vida y la salud. Si existe la posibilidad de exposición superior a 5000 ppm, utilice un equipo de respiración autónomo de máscara completa, aprobado por el NIOSH, en modo de presión a demanda u otro modo de presión positiva, con un cilindro de aire para escape de emergencia.

La exposición al metano es peligrosa ya que esta sustancia puede reemplazar el oxígeno y llevar a la asfixia. En atmósferas deficientes en oxígeno, solo utilice un equipo de respiración autónomo con máscara completa, aprobado por el NIOSH, en modo de presión positiva NJHEALTH, 2011).

En la presente investigación los niveles de concentración de metano en los recintos 1,2 y 3 oscilan entre $38 \mathrm{ppm}$ y $364 \mathrm{ppm}$, por lo que se concluye que dichos niveles no afectaran en la salud de las personas y que el aire que se respira es permisible.

\section{CONCLUSIONES}

En los núcleos seleccionados del centro experimental de Chachapoyas (establo), hay emisión de metano en bajos niveles.

La adecuada ventilación en los núcleos 1 y 3 , donde están ubicados los bovinos, permite que sea imperceptible al metano por parte de los trabajadores o del personal que presta servicio. La altura del techo es apropiada para la volatilidad del metano.

En el núcleo 2, donde se realiza el ordeñamiento de leche, es el que presenta mayor nivel de concentración de metano que está entre 70 ppm a 120 ppm. La mayor área de concentración de metano se localiza en el canal que se direcciona hacia el biodigestor, y en la salida del biodigestor donde se alcanza concentraciones de metano entre 700 ppm a 900 ppm.

Las excretas frescas de los bovinos, no generan metano; para que se produzca el metano debe considerarse los siguientes factores: temperatura ambiente, humedad, incidencia solar y el forraje que ingesta los bovinos.

En la presente investigación los niveles de concentración de metano en los núcleos 1, 2 y 3 oscilan entre $38 \mathrm{ppm}$ a $364 \mathrm{ppm}$, por lo que se concluye que dichos niveles no afectaran en la salud de las personas y que el aire que se respira es permisible, además los niveles de la concentración de metano durante el día y horas no son mantiene constante por la volatilidad del gas. La calidad de aire que se respira en los núcleos 1, 2 y 3 es saludable, dado que los niveles de metano son inferiores a los valores permitidos.

Los efectos de estacionalidad y de tendencia observados en los gráficos respecto a los niveles de metano, se debe principalmente a las corriente de aire, por lo que se puede indicar que el metano es más ligero que el aire.

\section{REFERENCIAS BIBLIOGRÁFICAS}

Albritton, D.L. y Meira, L.G. (2001). Technical Summary: A report accepted by Working Group I of the IPCC.

Bonilla Cárdenas, J. A., y Lemus Flores, C. (2012). Emisión de metano entérico por rumiantes y su contribución al calentamiento global y al cambio climático: Revisión. Revista mexicana de ciencias pecuarias, 3(2), 215-246.

Carmona, J. C., Bolívar, D. M., y Giraldo, L. A. (2005). El gas metano en la producción ganadera y alternativas para medir sus emisionesy aminorar su impacto a nivel ambiental y productivo. Revista ColombianadeCiencias Pecuarias, 18(1).

Chadwick, D. (2005). Emissions of ammonia, nitrous oxide and. Atmospheric Environment methane from cattle manure heaps: effects of compaction $a n d$ covering., 39:787-799.

Congreso de la República, S. (2014). Compendio Legislativo sobre el cambio climativo en el Perú. Lima: Sociedad Peruana de Derecho Ambiental.

Cuatecontzi, D.H. y Gasca, J. 2004. Los gases regulados por la convención marco de las naciones unidas sobre el cambio climático. En Cambio Climático una v is ión desde México. Compiladores: J u 1 i a Martínez y Adrián Fernández. SEMARNAT-INE.

Gómez, C. V. (2005). Emisión de metano y sistemas de producción animal en el P e rú: Implicancias Nutricionales. International Farm Comparison Network, Global Farm GbR, Braunschweig, , 143-144. 
IPCC (Intergovernmental Panel on Climate Change).2001. The third a s s e s s m e n t report, climate change 2001. Cambridge University Press, Cambridge, UK.

IPCC -Intergovernmental Panel on Clime Change-, 1996c. Directrices del IPCC para los inventarios de gases de efecto invernadero, versión revisada en 1996 , cuaderno de trabajo, volumen 2. Editores: J.T. Houghton, L.G. Meira Filho, B. Lim., K. Tréanton, I. M a m a ty, Y. Bonduki, D.J. Griggs and B . A . Callander. IPCC/OCDE/AIE

Johnson, K.A. and Johnson, D.E. 1995. Methane emissions from cattle. J Anim Sci, 73: 2483-2492.

Kurihara M, Magner T, McCrabb H, McCrabb G. (1999). Methane production and energy partition of cattle in the tropics. British Journal of Nutrition, 81:227-234.

Leganés, M. d. (30 de Enero de 2017). Ecologistas en acción. Obtenido de Contaminación del aire y la salud: http://www.ecologistasenaccion.org/spip.ph p?article 5682

McCrabb, G.J and Hunter, R.A. (1999). Prediction of methane emissions from beef cattle in tropical production systems. Aust. J . A g r i c . R e s. 50:1335-1339

Methane to Markets, (2008). La importancia el metano y las actividades de reducción de sus emisiones. Administrative Support Group Methane to Markets Partnership www.methanetomarkets.org. Marzo de 2008.

Misselbrook T.H., P. J. (2016). Emisión de amoniaco durante los procesos de compostaje y vermicompostaje: Aspectos prácticos y aplicados. Agroproductividad, 46-47.

Montenegro J, Abarca S. (2000). Fijación de carbono, emisión de metano y de óxido nitroso en sistemas de producción bovina en CostaRica. En: Intensificación de la ganadería en $\quad \mathrm{C} e \mathrm{nt} \mathrm{r}$ o a méri c a : beneficios económicos y ambientales. CATIE - $\quad$ FAO - SIDE. Ed Nuestra Tierra. $334 p$
MTS, S. (2012). Greenhouse gases and agriculture in Cambodia - GGAAC. Cntribución al calentamiento, Rev Mex Ciencia Pecuaria 2012 ;3(2): 215-246.

NJHEALTH, (2011). New Jersey Departament of Health. Methane. Traducción Marzo de 2012. Línea directa del NJ DEP: 1- 877 927-6337

Parkinson R., G. P. (2004). Effect of turning regime and seasonal weather conditions $\mathrm{o} \quad \mathrm{n}$ nitrogen and phosphorus losses during aerobic composting of cattle $\mathrm{m}$ a $\mathrm{n} \mathrm{u} \mathrm{r}$ e. Bioresource Technology, 91:171-178.

Pope1l CA, Buernett R, Thun M, et al. Lung (2002). Cancer, Cardiopulmonary Mortality, and Long-term Exposure to Fine Particulate Air Pollution. JAMA $\quad 2 \quad 0 \quad 0 \quad 0 \quad 2$; 287(9):1132-1141.

Primavesi O, Shiraishi RT, Dos Santos M, Aparecida M, Teresinha T, Franklin P. (2004). Metano entérico de bovinos leiteiros em condiçðes tropicais brasile ir as Pesqagropec bras, 39 (3): $\quad$ 277-283.

Rynk R., V. d. (1988). Northeast Regional Agricultural Engineering Service. On farm composting handbook, 4-5.

TESTO. (2013). Ingenieria de medición para prevención. DISAI, 2-5.

Tiquia S.M., T. N. (2000). Fate of nitrogen during composting of chicken litter. Environmental Pollution, 110:535-541.

UNAM. (03 de febrero de 2017). Calidad del aire.Obtenidodehttps://calidaddelaire.wordp ress.com/

USEPA. (20 de enero de 2005). National emissions inventory. Obtenido de United Sates Environmental Protection A ge n c y, Wa shington D.C.: http://www.epa.gov/ttn/chief/net2002invent ory.html

USEPA-Environmental Protection Agency- 1994 . International Anthropogenic Methane Emissions: Estimates for 1990 EPA230-R-93-010. Washington, D C : Office of Policy, Planning and Evaluation, USEPA.

Velasco-Velasco J., F.-S. B.-C. (2004). CO2 and Microbial Population Dynamics in Manure and Straw Compost under Aeration. Terra Latinoamericana, 22:307-316. 
Von Bernard, H., Vilarino, V., y Piñeiro, G. (2007).

Emisión teórica de metano en $\quad \mathrm{t} r$ e $\mathrm{s}$ sistemas de invernada para engorda $\mathrm{d} \quad \mathrm{e}$ ganado en Argentina. Ciencia $e$ investigación agraria, 34(2), 121-129. 